Wien klin Mag 2016 · 19:69-70

DOI 10.1007/s00740-016-0119-0

Online publiziert: 8. Juni 2016

(c) Springer-Verlag Wien 2016

CrossMark

\section{Karl Lhotta}

Abteilung für Nephrologie und Dialyse, Akademisches Lehrkrankenhaus Feldkirch, Feldkirch, Österreich

\title{
53. Kongress der Europäischen Nephrologischen Gesellschaft
}

\author{
"From big data to personalized therapy - \\ biostatistics meets molecular medicine ${ }^{\prime \prime}$
}

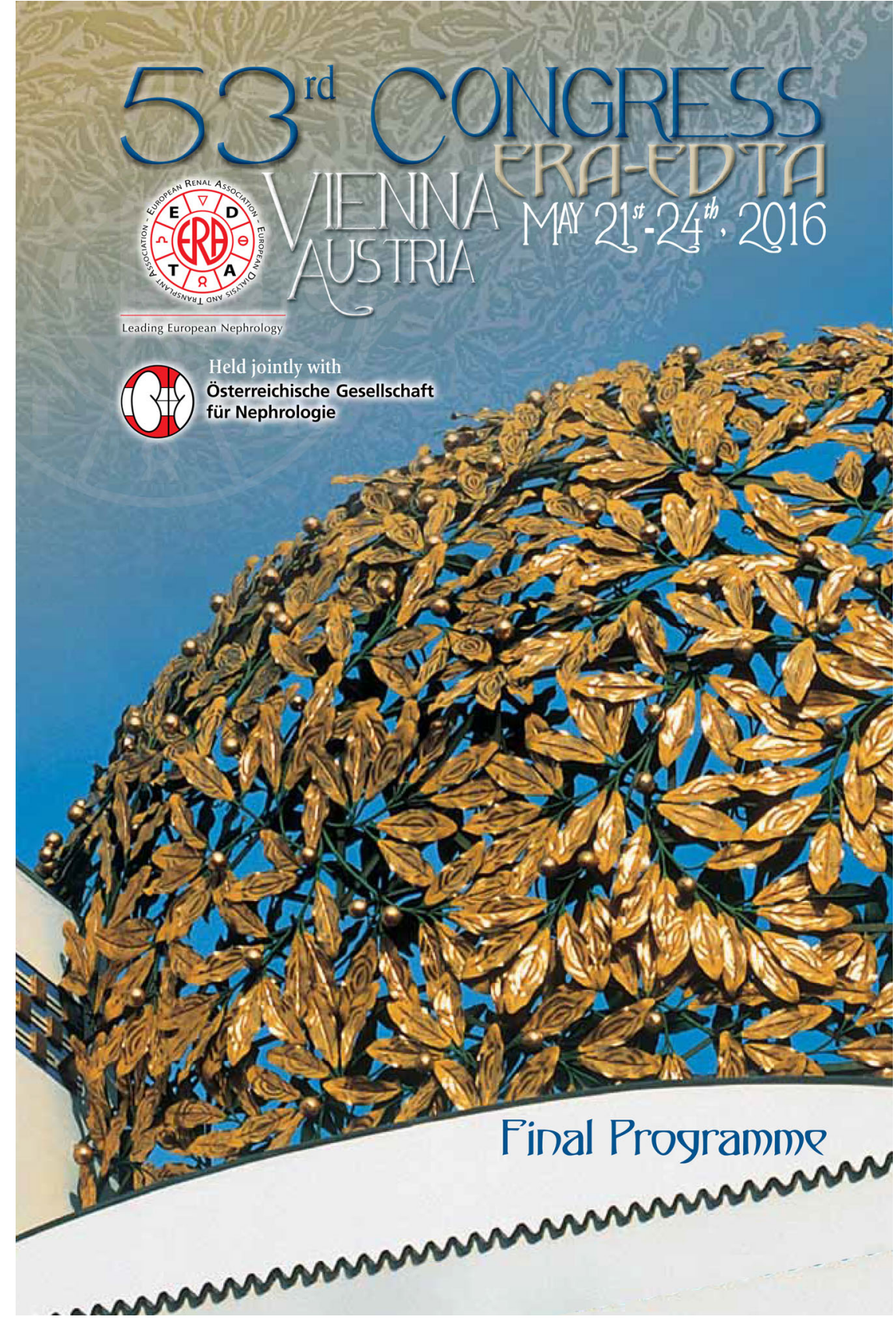

Das übergeordnete Motto des 53. Kongresses der Europäischen Nephrologischen Gesellschaft ERA-EDTA, der heuer von 21. bis 24. Mai in Wien stattfand, lautete „From big data to personalized therapy - biostatistics meets molecular medicine“. In enger Zusammenarbeit zwischen Kongresspräsident Gert Mayer und der Österreichischen Gesellschaft für Nephrologie organisiert, führte der Kongress mehr als 8000 Teilnehmer aus der ganzen Welt nach Wien. Wie in vielen anderen Gebieten in der Medizin entspricht die aktuelle Therapie nicht den Anforderungen einer personalisierten oder Präzisionsmedizin. Die number needed to treat für die Mehrzahl von Medikamenten zur Verhinderung eines harten Endpunktes ist nach wie vor viel $\mathrm{zu}$ hoch. Viele $\mathrm{Pa}$ tienten nehmen Medikamente ein, die ihnen nicht helfen und im schlimmsten Fall Nebenwirkungen verursachen. Die Kosten für die Gesundheitssysteme sind enorm. In seiner lecture Is it time for one person trials? entwarf Dr. Nicholas Schork ein Konzept, das weg von großen randomisierten Studien mit hohen Patientenzahlen zu Ein-Personen Studien, in denen auf die genetische Individualität und die Lebensumstände der Testperson eingegangen werden kann, führt.

Ein zweiter zentraler Aspekt des Kongresses waren Prävention und Früherkennung von Nierenerkrankungen. Raymond Vanholder aus Gent präsentierte die Empfehlungen der European Kindey Health Alliance, welche die Umsetzung dieser Ziele auf europäischer Ebene anstrebt. Dabei geht es vor allem um die Prävention chroni- 
scher Nierenerkrankungen und anderer Erkrankungen, die durch falsche Ernährung, Bewegungsmangel und Rauchen verursacht werden. Nierenerkrankungen lassen sich leicht und kostengünstig anhand der Bestimmung der errechneten glomerulären Filtrationsrate aus dem Serumkreatinin und der AlbuminKreatinin Ratio im Harn nachweisen. Diese Screening-Untersuchungen sollten bei allen Risikopatienten wie solchen mit Diabetes, Bluthochdruck, Adipositas und positiver Familienanamnese durchgeführt werden. Mindesten zehn Prozent der Bevölkerung sind von einer chronischen Nierenschädigung betroffen. Drei Viertel aller Patienten mit deutlich eingeschränkter Nierenfunktion sind sich ihrer Nierenerkrankung nicht bewusst. Dadurch werden therapeutische Möglichkeiten, ein Fortschreiten der Nierenerkrankung zu verhindern und das massive Risiko für kardiovaskuläre Ereignisse, das mit Nierenerkrankungen einhergeht, zu verringern, nicht genutzt.

Unter den wichtigen therapeutischen Innovationen in der Nephrologie sind vor allem Hemmer des renalen Glukosetransporters SGLT2 bei Typ-2 Diabetes und vor allem diabetischer Nephropathie erwähnenswert. Diese Medikamente senken durch renale Glukoseausscheidung nicht nur das HbAlc, sondern sie führen zu einer leichten Volumendepletion und Blutdrucksenkung. Die günstigen Effekte auf kardiovaskuläre Endpunkte wurden bereits publiziert. Noch größer scheint der Benefit dieser Substanzen auf das Neuauftreten oder Fortschreiten einer diabetischen Nierenerkrankung zu sein. Dies dürfte vor allem durch die Hemmung der glukoseabhängigen $\mathrm{Na}$ triumrückresorption im proximalen $\mathrm{Tu}$ bulus und damit Blockierung des tubuloglomeruären Feedbacks mit glomerulärer Hyperfiltration erklärt werden, ein Effekt, der demjenigen von ACE-Hemmern und Angiotensinrezeptorblockern ähnlich ist. Zusätzlich dürfte die eingeschränkte Glukoserückresorption interstitieller Inflammation und Fibrose entgegenwirken.

Erstmals scheinen auch große Fortschritte in der Therapie der autosomal dominanten polyzystischen Nierenerkrankung möglich. Die ADPKD betrifft
10 Prozent aller Patienten an der Nierenersatztherapie, ihre Prävalenz in der Bevölkerung liegt bei 1:2500. In der TEMPO 3:4 Studie konnte gezeigt werden, dass Tolvaptan, ein Hemmer des Vasopressinrezeptors an Tubulusepithelzellen, das Zystenwachstum und damit auch wahrscheinlich die Abnahme der Nierenfunktion günstig beeinflussen kann. Der Fokus aktueller Untersuchungen liegt darauf festzustellen, welche Patienten eine rasche Progression ihrer Erkrankung haben und daher, ganz im Sinne der Präzisionsmedizin, besonders von einer Therapie profitieren würden.

Eine weitere neue therapeutische Option wurde für die Therapie ANCAassoziierter Vaskulitiden mit milder Nierenbeteiligung berichtet. Bei diesen Erkrankungen kommt es auch zu einer Aktivierung des alternativen Komplementweges. Das dabei freigesetzte Anaphylatoxin C5a scheint dabei von besonderer Bedeutung für die Inflammation zu sein. In der CLEAR-Studie wurde die Effektivität des oralen C5a-Rezeptorblockers CCX168 in Kombination mit Cyclophosphamid oder Rituximab untersucht. CCX168 diente dabei als partieller oder kompletter Ersatz der Steroidkomponente. CCX168 war dabei mindestens so effektiv wie Steroide in der Induktion einer Remission der Erkrankung bei besserer Lebensqualität und geringerer Nebenwirkungsrate.

Die folgenden Beiträge können nur einen kleinen Aspekt des Faches Nephrologie darstellen, zeigen aber die Vielfalt und Komplexität dieses spannenden Teilgebietes der Inneren Medizin.

\section{Karl Lhotta}

\section{Korrespondenzadresse}

\section{Prim. Prof. Dr. K. Lhotta}

Abteilung für Nephrologie und Dialyse, Akademisches Lehrkrankenhaus Feldkirch Carinagasse 47, 6800 Feldkirch, Österreich Karl.Lhotta@lkhf.at

Interessenkonflikt. K. Lhotta gibt an, dass kein Interessenkonflikt besteht. 